\title{
A metric to quantify virtual scene movement for the study of cybersickness: definition, implementation, and verification
}

Richard H.Y. So*, Andy Ho, and W.T. Lo

Human Performance and Virtual Reality Laboratory

Department of Industrial Engineering and Engineering Management

Hong Kong University of Science and Technology

Clear Water Bay, Kowloon, Hong Kong.

* corresponding author: rhyso@ust.hk

\section{ABSTRACT}

This paper presents a metric to quantify visual scene movement perceived inside a virtual environment (VE) and illustrates how this method could be used in future studies to determine a cybersickness dose value to predict levels of cybersickness in VEs. 'Sensory conflict theories' predict that cybersickness produced by a VE is a kind of visually induced motion sickness. A comprehensive review indicates that there is only one subjective measure to quantify visual stimuli presented inside a VE. A metric, referred to as 'spatial velocity (SV)', is proposed. It combines objective measures of scene complexity and scene movement velocity. The theoretical basis for the proposed SV metric and the algorithms for its implementation are presented. Data from two previous experiments on cybersickness were re-analyzed using the metric. Results showed that increasing 'SV' by either increasing the scene complexity or scene velocity significantly increased the rated level of cybersickness. A strong correlation between 'SV' and the level of cybersickness was found. The use of the 'spatial velocity' metric to predict levels of cybersickness is also discussed.

Key Words: Cybersickness, Spatial frequency, vection, virtual reality, virtual environment 


\section{INTRODUCTION}

\subsection{Background}

Motion sickness with virtual environments (VEs) has been referred to as cybersickness (McCauley and Sharkey, 1992) and has been the subject of many studies. A review of literature indicates that the effects of variables related to the tasks, apparatus, and participants as well as methods to measure cybersickness have been studied. Examples of task-related variables that have been studied include duration of exposure (e.g., Cobb, Nichols, Ramsey and Wilson, 1999; Stanney and Kennedy, 1998), habituation (e.g.,Howarth and Hill, 1999; Regan, 1995), recovery period (e.g., Stanney, Kennedy, Drexler and Harm, 1999), navigation velocities (So, Lo and Ho, 2000b), methods of navigation (e.g., Howarth and Finch, 1999; Stanney and Hash, 1998), and viewing posture (e.g., Ehrlich, Singer and Allen, 1998; Regan, 1995). Factors related to the apparatus and participants have also been studied. Examples include display's field-of-view (e.g., Dizio and Lackner, 1997), types of display: (e.g., Cobb et al., 1999; Howarth and Costello, 1997), use of stereoscopic presentation (e.g., Ehrlich, 1997; Mon-Williams, Plooy, BurgessLimerick, and Wann, 1998), inter-pupillary distance mismatch (e.g., Regan, 1995), image update lags (e.g., Dizio and Lackner, 1997; Regan, 1995; So, 1994), gender, age, and pre-exposure stability (e.g., Kolasinski, 1995; Kolasinski and Gilson, 1999), and drug treatment (e.g., Regan, 1995).

A review of these studies indicates that there has been both agreement and disagreement among the reviewed studies. Examples of agreement include those studies on the effects of exposure duration - many studies have confirmed that moving in a VE for more than ten minutes will cause significant increases in nausea ratings (e.g., So, 1994; Regan, 1995; Howarth and Costello, 1997; Stanney and 
Kennedy, 1998; Stanney and Kennedy et al., 1999; Wilson, 1996; Wilson, Nichols and Haldane, 1997). Examples of disagreement include those studies on the effects of time lags - So (1994) reported that an imposed lag of $280 \mathrm{~ms}$ did not significantly affect the nausea ratings generated by a 20 -minute virtual flight simulation session. Although this finding agrees with the results reported by Regan (1995), it disagrees with the findings in Dizio and Lackner (1997). Regan reported that there was no significant difference in malaise ratings when the effects of updated lag were maximized by increasing head movements and speed of interactions while Dizio and Lackner reported that sickness severity increased monotonically with increasing lags up to 200ms. The differences in the results reported by So (1994) and Dizio and Lackner (1997) could be due to differences in factors such as the visual content of the VE, the nature of the tasks, or types and speeds of head movements. For example, the head movements reported by in So (1994) were slow $(0.02 \mathrm{~Hz}$ oscillation with an amplitude of $24^{\circ}$ r.m.s.) and continuous (sinusoidal motion) while the head movements of Dizio and Lackner (1997) were discrete with large amplitudes $\left(18^{\circ}\right.$ to $\left.180^{\circ}\right)$.

This example illustrates the difficulty in comparing results of cybersickness studies that use different virtual scenes and tasks. One possible solution to reduce this problem would be to identify a metric for quantifying the visual scene movement that a user is exposed to inside a VE. Furthermore, a review of literature identifies that 40 out of 50 studies on cybersickness have used the Simulator Sickness Questionnaire (SSQ, Kennedy, Lane, Berbasum and Lilenthal, 1993) to measure the levels of cybersickness. While the commonly used SSQ has facilitated the comparison of sickness responses among studies, it is a unfortunate that there is as yet no common method to quantify the scene movement exposure among different experiments. 


\subsection{Importance of visual stimuli}

The use of the sensory conflict theory (Reason and Brand, 1975), the sensory rearrangement theory (Reason, 1978), and the motion cue mismatch theory (Benson, 1984) as explanations for cybersickness has been widely discussed (e.g., Cobb and Nichols et al., 1999; Hettinger and Ricco, 1992; Oman, 1993, Regan, 1995; Kennedy, Lanham, Drexler, Massey and Lilienthal, 1995, McCauley and Sharkey, 1992; So, 1994; Stanney and Salvendy et al., 1998). Reason and Brand (1975) and Reason (1978) proposed the sensory-rearrangement theory, which predicts that when two or more sensory cues disagree or at variance with the expected experience, symptoms of sickness can occur. As pointed out by Oman (1993): "no sensory conflict neuron or process has yet been physiologically identified". Sensory conflict, therefore, remains a hypothetical parameter which cannot be measured directly. As a consequence, sensory conflict theory cannot be proved or disproved through direct measurements. A similar conclusion was reached by Griffin (1990) who searched for a theory to explain seasickness. In his book, Griffin (1990) presented the benefits of

studying the conflict of sensory 'stimuli' rather than the conflict of senses. The former can be measured readily while the latter cannot be measured. This is consistent with the shift of research focus from sensory mismatch to motion cue mismatch as suggested by Benson (1984). The 'observer theory model' on sensory conflict proposed by Oman $(1982,1993)$ also focuses on stimulus conflicts rather than on sensory conflicts. Cybersickness experiments should, therefore, focus on the relationship between the relevant sensory stimuli and their associated sickness levels and symptoms. In the case of VR simulation, one of the major sensory stimuli to the users will be the visual stimulus.

When an observer views a wide field-of-view moving scene, illusory selfmotion (vection) in the direction opposite to the moving scene may be generated 
(Griffin, 1990). This experience can be nauseogenic. A common conclusion found in cybersickness literature is that cybersickness is mainly due to this visually induced self-motion illusion (vection) which is inappropriate to the physical motion of the participant (e.g., Hettinger and Ricco, 1992; McCauley and Sharkey, 1992; Regan, 1995). This type of motion-cue mismatch has been identified previously in studies of 'Cinerama sickness' and 'Simulator sickness' (Benson, 1984). In the case of cybersickness, participants may receive visual cues to induce an illusion of body motion while their bodies are physically stationary. This causes discord between their visual and vestibular cues and, hence, sickness. The occurrence of vection in stationary participants has been associated with symptoms of nausea and sickness in many studies (e.g., studies with VR systems: Cobb et al.,1999, McCauley and Sharkey, 1992; studies with an optokinetic drum: Dichgans and Brandt, 1978, Kennedy, Hettinger, Harm, Ordy and Dunlap, 1996; Stern, Hu, Leblanc and Koch, 1993; studies with a non-VR simulator: Dizio and Lackner, 1991, Kennedy and Frank, 1984). Although these studies used different apparatus to present visual cues to the participants, they have one common characteristic - participants were exposed to visual scene movements.

Consequently, scene movement may be an important contributing factor in cybersickness and the ability to measure and quantify scene movements is an important and interesting research topic. Such research is also consistent with the effort to identify and prioritize the sensory-motor discord that drive cybersickness - a research direction proposed by 26 leading experts in the field of cybersickness research (Stanney and Slavendy et al., 1998).

\subsection{Previous studies on measuring and quantifying scene movements for the study of cybersickness}

A review of literature reveals only four studies which have investigated the effects of 
scene movements on the level of cybersickness. Slater, Linakis, Usoh and Kooper (1996) asked the participants to perform manual manipulation tasks (chess games) in VEs with different textural backgrounds. Both performance and sickness were measured. Unfortunately, the sickness data were not reported. Prothero, Draper, Furness, Parker and Wells (1999) demonstrated that viewing a rotating scene $\left(25^{\circ}\right.$ horizontal $\times 19^{\circ}$ vertical) projected on a dark background produced significantly higher levels of cybersickness than viewing the same rotating scene augmented on top of normal vision. In the latter case, the ambient field-of-view corresponded appropriately with the participants' head orientation. Prothero and his colleagues attributed the results to the theory of the 'rest frame'. Unfortunately, there was no report of an objective method to identify and quantify a 'rest frame' within a VE.

Kennedy, Berbaum and Smith (1993) attempted to quantify visual features within a flight simulator scene that were correlated with the level of simulator sickness. In their studies, visual features (e.g., position of the horizon) were identified through visual inspection. In 1996, Kennedy and his colleagues proposed an automatic method to quantify the visual stimulus for cybersickness (Kennedy and Berbaum et al., 1996). In their studies, simulation scenes were videotaped and viewed by a human observer who would then assign scores to the scenes. Cluster analyses were then conducted on the scores and the resulting metrics for quantifying the visual stimulus were referred to as the "human judged kinematics cluster score (HJKCS)'. This work by Kennedy represented an important step towards quantifying the visual stimulus in the study of cybersickness. Unfortunately, the proposed 'human judged kinematics cluster score' is subjective and its compilation is labor intensive. Currently, there is still no point-fine objective metric for quantifying the visual information viewed in a VE.

It should be noted that although only a few studies have attempted to quantify scene movement in a VE for the study of cybersickness, there have been decades of studies in search of a quantitative metric for optical flow information and its 
relationship with the perception of self-motion in simulators (e.g., Gibson, 1947, 1950; Gibson, Olum and Rosenblatt, 1955; Owen and Warren, 1982, 1987). In addition, there have been many publications in the field of neuro-physiology on the understanding of vection generation (e.g., the 'two-visual system': Schneider, 1967; Leibowitz and Dichgans, 1980; Leibowitz and Post, 1982). Furthermore, studies concerning vection-induced motion sickness with rotating optokinetic drums have also been many (e.g., Stern and Koch, 1991; Hu, Stern, Vasey and Koch, 1989; Hu et al., 1997; Kennedy, Hettinger, Harm, Ordy and Dunlap, 1996). These studies are reviewed in Sections 2.1 amd 2.2 to form a theoretical basis for the proposed 'spatial velocity' metric.

\section{THE PROPOSED METRIC FOR QUANTIFYING SCENE MOVEMENT IN A VE}

\subsection{The proposed 'spatial velocity' and its theoretical basis}

\subsubsection{Definition of the proposed 'spatial velocity' metric}

In this work, a metric called 'spatial velocity (SV)' was developed to quantify visual stimuli for the study of cybersickness. This proposed SV metric has two components: (i) a metric to measure the scene complexity of a VE along horizontal, vertical, and radial axes; and (ii) a metric to measure the velocities of navigation by the participant through the VE in fore-and-aft, lateral, vertical, pitch, yaw, and roll directions. These two components are multiplied to form the rate of luminance variation perceived by a participant when he or she navigates through a VE:

\footnotetext{
The rate $=$ (average spatial frequency, SF, along that axis) $\mathrm{X}$ (scene movement velocity, $\mathrm{V}$, along that axis)
}

This rate is referred to as the 'spatial velocity' (SV) for that axis. It combines the 
scene complexity of a visual scene (SF) and it's movement $(\mathrm{V})$ relative to the participant. The main differences between the proposed SV metric and the previously reported HJKCS (Kennedy and Barbaum et al., 1996) are (i) the HJKCS involves subjective judgement while the SV metric is totally objective; (ii) the calculation of HJKCS is labor intensive while the SV metric can be calculated by computer programs. Since scene movements are common among VR simulation, the SV metric is a generic measure not restricted to a particular application. Spatial velocities for all six motions (x-fore-and-aft, y-lateral, z-vertical, yaw, roll, and pitch) are calculated as:

$$
\left[\begin{array}{ll}
S V_{x} & S V_{\text {roll }} \\
S V_{y} & S V_{\text {yaw }} \\
S V_{z} & S V_{\text {pitch }}
\end{array}\right]=\left[\begin{array}{lll}
S F_{\text {rad }} & S F_{\text {horiz }} & S F_{\text {vert }}
\end{array}\right] \times\left[\begin{array}{ll}
V_{x} & V_{\text {roll }} \\
V_{y} & V_{\text {yaw }} \\
V_{z} & V_{\text {pitch }}
\end{array}\right]
$$

where $\mathrm{SV}_{\mathrm{x}}, \mathrm{y}, \mathrm{z}$, yaw, roll, pitch are the spatial velocities of the fore-and-aft, lateral, vertical,

\author{
yaw, roll, and pitch motions; \\ $\mathrm{SF}_{\text {horiz, rad, vert, }}$ are the spatial frequencies of scene components along the \\ horizontal, radial, and vertical axes; \\ $V_{x, y, z, y a w}$ roll, pitch are the scene movement velocities along the six axes.
}

The logic of mapping the appropriate spatial frequencies (SF) to the scene movement velocities $(\mathrm{V})$ is straightforward except for the case of $\mathrm{SV}_{\mathrm{x}}$. It is mapped to the radial spatial frequency $\left(\mathrm{SF}_{\mathrm{rad}}\right)$ because the radial dimension of 'virtual objects' in the scene will change according to the fore-and-aft movement of the scene relative to the subject. This is analogous to shining a torch at a screen - as the torch moves towards the screen, the light spot will change its radial dimension.

Details on the development, measurement, and implementation of the SV 
metric are described in Sections 2.2 to 2.5. In the rest of this section, literature concerning the effects of optical flow elements on the perception of self-motion in non-VR systems is reviewed to form the theoretical basis for the two-component structure of the SV metric.

\subsubsection{Theoretical supports}

In Section 1.2, the close association between illusory self-motion (i.e., vection) and cybersickness has been discussed. It has been reported that vection is a necessary precondition for simulator sickness (Hettinger, Berbaum, Kennedy, Dunlap and Nolan, 1990) and cybersickness (Hettinger and Riccio, 1992). In the search for a measure to quantify scene movement for the study of cybersickness, it is logical to review literature on the self-motion illusion (i.e., vection).

The perception of self-motion as a result of viewing scene movement has been the subject of many classic studies concerning flight simulation training (e.g., Gibson, 1947). In particular, Gibson et al. (1955) quantified the optical flow patterns of visual objects perceived by a pilot during a flight simulation session. The flow patterns were represented by a function consisting of the relative velocities between the objects of interest and the aircraft. The effects of these flow patterns and other optical variables on the perception of self-motion have subsequently been studied extensively (e.g., Owen and Freeman, 1986; Owen and Jensen, 1981; Owen, Warren, Jensen, Mangold and Hettinger, 1981; Owen, Wolpert and Warren, 1984; Owen, Hettinger, Pallos and Fogt, 1985; Owen and Warren, 1987). The variables investigated include the optical flow rate (flight velocity divided by instantaneous altitude); optical acceleration (product of flight velocity and descent rate divided by the square of the difference between the initial altitude and altitude to be descended to); optical density (instantaneous altitude divided by ground texture size); and optical splay (rate of descent divided by altitude).

As reported by Owen and Warren (1987), these optical variables have been 
specific to flight simulation. In an attempt to generalize the measurement of optical flow, another two optical variables have been proposed: (i) the optical flow rate accelerations (FRAs) in the fore-and-aft and vertical directions and (ii) the edge rate acceleration (ERA) along the flight path. Owen and Warren (1987) used an analogy with road traffic to explain optical flow rate and edge rate: "Flow rate is analogous to measuring the speed at which vehicles pass (e.g., in km per hour), whereas edge rate is analogous to measuring the frequency with which vehicles pass (e.g., in number per hour)" (quoted from Owen and Warren, 1987). In Owen's study, the optical FRA represents the perceived acceleration (unit distance / second ${ }^{2}$ ) of a participant who is navigating through a flight simulation environment while the ERA represents the perceived acceleration in the number of contrasted edges on the ground that a participant will pass per unit time (e.g., number of edges per second ${ }^{2}$ ). In other words, the FRA is a pure acceleration measurement that is independent of scene complexity while the ERA combines both the navigation speed as well as the scene complexity of the ground texture. Owen and Warren (1987) reported that ERA has a stronger influence on the perception of self-motion than optical FRA. This suggests that ERA may be a better variable to describe optical flow than FRA. Comparing the ERA proposed by Owen and Warren (1987) with the proposed spatial velocity variable (SV):

- $\quad$ Both the proposed SV and ERA have similar components: (i) navigation speed or its derivative, and (ii) complexity of scene texture.

- SV considers speeds of navigation in all six motions while the ERA considers only speed of navigation in the fore-and-aft direction (ERA focuses on the fore-and-aft axis because that is the main axis of movement for an aircraft).

- In ERA, scene complexity is measured using a unit customized for simulation environments with square-textured ground, while in SV scene complexity is measured by a generic unit of 'spatial frequency' (more details in Section 
In short, ERA and SV are made up of similar components but SV is a more generic metric while ERA has been developed for the flight simulation environment.

Hettinger, Owen and Warren (1985) studied the perception of vection with different ERAs. In their study, the flight velocity was kept constant and the ERA was increased by increasing the density of the edges (i.e., reducing the size of the squared texture on the ground so as to increase the number of edges per unit distance). The results indicated that as the ERA increased, the perception of vection increased, peaked, and then reduced. In 1989, Hu et al. reported a study investigating the level of vection generated by participants viewing an optokinetic drum rotating at different speeds. The results indicated that as the speed increased, the level of vection increased, peaked, and then reduced - a pattern similar to that of Hettinger et al. (1985). Kennedy, Hettinger, Harm, Ordy and Dunlap (1996) also reported that as the speed of optokinetic drum increased, the latencies for circular vection (CV) to occur reduced, bottomed, and then increased. These latencies have been recognized to inversely relate to the level of motion sickness. The agreement among these three studies suggests that an increase in scene velocity (reported in Hu et al., 1989 and Kennedy and Hettinger et al., 1996) may have an effect on the level of vection similar to that of an increase in scene complexity (reported in Hettinger et al., 1985). This supports the multiplication of the scene complexity term and the scene velocity term in the SV metric. In conclusion, the proposed SV metric is consistent with the previous literature on optical flow measurement (e.g., Owen and Warren, 1987), self motion perception in flight simulation (e.g., Hettinger et al., 1985) and vection generation with an optokinetic drum (e.g., Hu et al., 1989).

\subsection{Methods to measure scene complexity}

\subsubsection{The proposed method to measure scene complexity}


In this study, the 'scene complexity' of a VE during a simulation session was measured by averaging the scene complexities of sampled snap shots taken during the simulation. The scene complexity of each snap shot was measured by three descriptions: the average spatial frequencies (SF) along the horizontal, vertical, and radial axes. Details on how to measure SF is documented in Section 2.2.3. The average SFs along the horizontal and vertical axes were the means of the SFs across all pixel rows and columns, respectively. The average SF along the radial axis was the geometric mean of the average SFs along the horizontal and vertical axes. For example, if a snap shot had 640 columns and 480 rows of pixels, the SF along each of the 640 columns was measured and the mean of these 640 dominant SFs was the average SF along the vertical axis of that snap shot. Similarly, the mean of the 480 SFs along each row was the average SF along the horizontal axis. Having stated the methods to measure 'scene complexity' of a VE, three questions remain: (i) why was spatial frequency (SF) used? (ii) how was the SF of a row or column of pixels determined? and (iii) how was the number of sampled snap shots determined? Sections 2.2.2 to 2.2.4 will address these three questions.

\subsubsection{Rationale for using spatial frequency to measure scene complexity}

In this work, scene complexity was divided into two sub-components: (i) a luminance component, and (ii) a chrominance component. These two components are the typical sub-components of a television picture signal. The luminance component represents the spatial distribution of luminance intensity while the chrominance component represents the spatial distribution of the color. The search for a suitable measure to quantify scene complexity for the study of cybersickness focussed on these two aspects. It should be noted that the 'meaning' aspect of the scene was purposely omitted. Although Kennedy, Berbaum and Smith (1993) reported that the position of some specific visual elements such as the artificial horizon may influence the level of simulator sickness, the identification of such elements is application 
specific and can be subjective. Since the proposed SV is designated to be an objective generic metric, the subjective meaning behind the scene was not considered in the proposed SV metric.

In a study of visually induced linear vection with a simulated aerial view of a textured ground (random dot pattern), Previc, Varner and Gillingham (1992) reported that neither color (instead of black-and-white graphics) nor perspective view affected the level of perceived vection. This suggests that the chrominance component of a scene may not have a strong influence on vection. In a literature review concerning cybersickness, Kolasinski (1995) also hypothesized that color would not have a direct effect on the level of cybersickness, although it might influence the level of graphical realism. The reported reason was that people use peripheral vision to detect movement and peripheral vision is poorly sensitive to color. This agrees with Livingstone and Hubel (1988) who reported that there is consistent evidence in both physiological and psychophysical studies to show that the visual system that is responsible for movement perception is not sensitive to color and is also not used for shape discrimination. Without intending to rule out the influence of the chrominance component of a scene on the level of vection that can be generated, as an initial implementation, scene complexity was measured by its luminance component only. Future studies to compare VEs with isochronal or isolumiant colored mappings are desirable.

Previous work by Owen and his colleagues on quantifying optical flow measured the luminance density of scene complexity by counting the number of contrasted edges per unit distance (Owen and Warren, 1987; see reviews in Section 2.1). In Owen's studies, the counting was possible as the scene of interest was a simulated ground with squared texture and the edge density was simply worked out from the size of the squares (e.g., Tobias and Owen, 1984; Hettinger et al., 1985). The SV metric proposed here is intended to be applicable in environments containing different textures. Consequently, a more generic method of measuring luminance 
density is needed.

A review of the literature indicates that in many studies concerning the visually induced self-motion illusion (vection), the patterns of luminance variations have been quantified in terms of 'spatial frequency' (e.g., Brandt, 1973; Dichgans and Brandt, 1978; Henn, Cohen and Young, 1980; Hu et al., 1989, 1997; Koch and Stern et al., 1990; Stern, Hu, Vassey and Koch, 1989; and Muller, Wiest and Deecks, 1990). The unit for spatial frequency (SF) is defined as 'cycles of changes in luminance per unit visual angle' or 'cycles per degree' (cpd). A cycle refers to a complete cycle of luminance variation. Experimental results have shown that approximately $50 \%$ of healthy participants manifest nausea symptoms when exposed to rotating black-and-white stripes with a horizontal SF of 1/15 cpd (e.g., Stern, Koch, Leibowtz, Shupart and Stewart, 1985; Stern and Koch, 1991). Hu et al. (1997) studied the level of vection and sickness as functions of SFs. Hu varied the SFs of black-and-white stripes inside an optokinetic drum from 1/60 cpd to 16/60 cpd and reported that the level of vection and sickness increased, peaked, and then reduced. This suggests that the SFs of a moving scene have a significant influence on the level of vection and sickness. Hu et al. (1997) found that the level of vection peaked at 4/60 (i.e., 1/15) cpd - a level of horizontal SF most often used in studies involving the optokinetic drums (e.g., Stern et al., 1985; Stern and Koch, 1991). In the SV metric, SFs along the horizontal, vertical, and radial axes were used to measure scene complexities.

Spatial frequency (SF) measures the frequency of spatial variations in luminance within a scene. However, it is an average value and does not indicate the exact details of shapes and patterns. In other words, a scene full of circular objects can have the same SFs as a scene full of rectangular objects. In 1990, Muller, Wiest and Deecks studied the level of vection generated from viewing a moving scene of different patterns: black-and-white stripes, random dots, and a checkerboard pattern of similar spatial densities. Results showed that there was no significant difference 
among the levels of vection obtained from viewing the three patterns. This suggests that the shapes of patterns may not be important to the induced vection. In addition, Leibowitz, Shupert-Rodemer and Dichgans (1979) reported that diopter refractive errors as large as those correspond to legal blindness have no influence on vection generation. Lebowitz and Post (1982) concluded that changes to the fine details of patterns have little influence on the associated vection.

In conclusion, reviews of the literature indicate that among luminance variation, chrominance variation, shapes, and patterns of a moving scene, the spatial variation of the luminance levels has the most influence on the level of vection generated. While spatial variation in the luminance level has been measured using spatial frequency (SF) as well as using contrasted edges per unit distance, SF is a more generic metric. Consequently, scene complexity in the SV metric was measured using SF.

2.2.3 Methods to determine the SF across a row or column of a snap shot Having decided to measure the scene complexity of a VE by averaging the dominant SFs of the rows and columns of sampled snap shots, a question remains as to how to determine the dominant SF of a row or column of a snap shot. A row or column of a snap shot is likely to contain luminance variations at different SFs. The standard method to extract the distribution of luminance variation as a function of SF is to calculate a spatial frequency power spectral density (SFPSD). Standard spectral analysis algorithms such as the fast fourier transform (FFT) can be used (Bendat and Piersol, 1986). The use of FFTs to calculate a SFPSD has long been a standard method in image processing (e.g., Schalkoff, 1989). However, there is no standard method to determine the dominant SF from a SFPSD. This study applied three methods to calculate the dominant SFs: (i) the mean method, (ii) the mode method, and (iii) the combined method. The mean method calculates the average SF according to the PSD distribution, while the mode method calculates the SF at which 
the amount of luminance variation peaked. The combined method calculates the dominant SF according to the following equation:

Dominant SF $=$ Average of all frequencies at which the SFPSD $>1 / 2(\text { SFPSD })_{\max }$

All three methods were used to re-analyze data from two previously reported experiments. The results are documented in Section 3.4.

\subsubsection{The number of sampled snap shots to be taken}

The purpose of taking sampled snap shots is to estimate the 'average' scene complexity of the whole VE as perceived by a user during a typical navigation. As with any sampling problem, an optimized number of samples is a compromise between the level of statistical accuracy and the resources available. Since the development of the proposed SV is still in its initial stage and the aim of this study is to determine the benefits of SV rather than optimizing the implementation of SV measurement, over-sampling was used. Future research to optimize the number of samples is proposed in Section 5.

In this study, sampled snap shots were taken twice per second over a simulation session of 30 minutes, yielding 3600 snap shots. Fortunately, the calculation of the proposed SV has been computerized. In the two experiments whose data were reanalyzed in Section 3, the navigation velocities have most of their energy below $0.4 \mathrm{~Hz}$. For navigation velocities with frequencies below $0.4 \mathrm{~Hz}$, a sampling rate of 2 snap shots per second (i.e., $2 \mathrm{~Hz}$ ) is well above the Nyquist sampling frequency of $0.8 \mathrm{~Hz}$ (i.e., $0.4 \mathrm{~Hz} \times 2$ ). Hence, over-sampling was employed (Oppenheim, Willsky and Nawab, 1997).

\subsection{Implementations to measure 'spatial frequency'}


With a specific task, most VR training applications have a typical navigation path; examples include a recommended flight path in flight simulation training (e.g., Wells, Venturino and Osgood, 1989) and a predetermined route in driving simulation (e.g., Bayarri, Fernandez and Perz, 1996). A program to capture views along the path at a rate of 2 snap shots per second was written. The captured pictures were stored in 'Portable Grey-Map', pgm, format so that the grey scales of all the pixel elements could be extracted as a matrix ( 255 is white and 0 is black). The 'pgm' format is supported by common graphics programs such as ' $x V^{\prime}$ in UNIX (or IRIS) and 'Paintshop-Pro' in WIN98, and the grey-scale matrix can be viewed as a text file. The grey scale value of each pixel element represents its luminance level.

Figure 1 illustrates the measurement of the dominant SF of a row within a visual scene. Inspection of the figure shows that the luminance information (i.e., grey scale) of each pixel in the row is extracted as a series of numbers, the power spectral density (PSD) of this grey scale series is then calculated using the FFT operation available in common signal processing software (e.g., MATLab, PC-DAT) (Bendat and Piersol, 1986). The result is the SFPSD. From the SFPSD, the average dominant frequency is determined by the three methods as explained in Section 2.2.3. The unit of the dominant SF is the number of cycle changes in luminance level per degree (cpd). This process is repeated for every row in the captured scene and the dominant SF of all rows are averaged to give the average horizontal spatial frequency $\left(\mathrm{SF}_{\text {horiz }}\right)$. Similarly, the average vertical spatial frequency $\left(\mathrm{SF}_{\text {vert }}\right)$ can be obtained. The radial spatial frequency $\left(\mathrm{SF}_{\mathrm{rad}}\right)$ is defined as the geometrical sum of $\mathrm{SF}_{\text {horiz }}$ and $\mathrm{SF}_{\text {vert }}$ (i.e., $\left.\mathrm{SF}_{\text {rad }}=\left[\mathrm{SF}_{\text {horiz }}{ }^{2}+\mathrm{SF}_{\text {vert }}{ }^{2}\right]^{1 / 2}\right)$. 
As illustrated in Figure 1, the power spectral density method was used to determine the dominant spatial frequency along a row or column of a captured scene (Bendat and Piersol, 1986). A possible alternative approach is to use the Michelson contrast $\left(C_{m}\right)$ instead of spatial frequency (Boff and Lincoln, 1988). This will involve the calculation of the Michelson contrast $\left(\mathrm{C}_{\mathrm{m}}\right)$ for each row and column of a captured scene (modified from Boff and Lincoln, 1988):

$$
C_{m}=\left(G_{\max }-G_{\min }\right) /\left(G_{\max }+G_{\min }\right)
$$

where $\quad G_{\max }$ is the maximum Grey-scale value along a row or column $G_{\min }$ is the minimum Grey-scale value along a row or column

Although the Michelson Contrast (MC) approach requires much less calculation than the spatial frequency (SF) approach, the later was chosen because $\mathrm{MC}$ only accounts for the two pixels with the maximum and the minimum grey-scale value along a row or column. Unlike the MC, the SF accounts for the frequency of variation and amplitude of the grey-scale values of all the pixels along a row or column. Consequently, SF was considered to provide more information than MC and was used in this study. A series of simulations was conducted to determine the effects of different windowing (e.g., Hanning, Hamming, or rectangular) and the length of FFT (e.g., 64, 128, 256) on the accuracy of a SFPSD. Results indicated that using a rectangular window introduced distortion and both Hanning and Hamming produced similar SFPSDs. With a display resolution of $640 \times 480$ and a field-of-view of $48^{\circ} \times 33^{\circ}$, an FFT length of 256 was used to produce a spatial frequency resolution of $0.05 \mathrm{cpd}$. Figure 2 illustrates the spatial frequencies of two different pictures. The first one is a pattern of black-and-white stripes while the second one is a snap shot taken in a VE used in a previous study on cybersickness (So, Lo and Ho, 2000a). 
[Insert Figure 2 about here]

\subsection{Implementation to measure the scene movement velocity (V)}

In a typical VE, the scene presented on a display is a view of the VE from a certain viewpoint. This viewpoint acts as a 'virtual' video camera which captures and projects images of 'virtual' 3D objects onto a 2D screen. The pointing direction of this viewpoint can be pre-programmed as well as controlled, in real-time, by the head orientation and navigation devices (e.g., a joystick). This viewpoint is usually represented within a VE simulation program as a vector containing $x, y, z$ position coordinates and yaw, roll, and pitch orientations. As this viewpoint coordinate changes, the scene presented on the display will change accordingly. By recording these viewpoint coordinates in a file, the scene movement displacement time histories in all six axes can be measured. The displacement time histories can then be differentiated to obtain the time histories of the velocities (relative to the user) from which the r.m.s. scene velocities in six axes can be calculated. The r.m.s. velocities are referred to as the scene movement velocities $(\mathrm{V})$. The coordinate system used to measure the direction component of navigation velocities is adapted from a previous study (So, Lo and Ho, 2000b). The coordinate system is called the 'viewer-centered coordinate' and defines the fore-and-aft, lateral, vertical, pitch, yaw, and roll directions with respect to the instantaneous pointing-direction of the participant's head.

Navigation data have been successfully extracted with a number of popular VE development programs including World-Tool-Kit (WTK) from Sense8, dVISE from DIVISION, and RENDERWARE from Critierion. All of these software packages have built-in functions to extract and record the viewpoint coordinates (e.g., WTK: 
'WTviewpoint_getlastposition'; 'WTviewpoint_getlastorientation', dVISE:

'VCBody_GetData'; 'VCTracker_GetData', RENDERWARE:

'RwGetCameraPosition'; 'RwGetCameraLTM').

\section{VERIFYING THE PROPOSED METRIC}

\subsection{Objective and hypothesis}

Data from two previously reported experiments were re-analyzed to determine the effects of SV on the rated level of cybersickness. The aim was to demonstrate that SV significantly correlates with the level of cybersickness. The two previous experiments had been conducted before the development of the proposed SV metric and their original aims were to investigate the effects of (i) scene velocity, and (ii) scene complexity on cybersickness (So, Lo and Ho, 2000a). The current objectives of the re-analyses of those data were to identify any significant relationships between (i) nausea ratings and spatial velocity; and (ii) SSQ total severity scores and SV. In addition, the three methods of calculating the dominant SF were compared. According to Hettinger et al. (1985) and Hu et al. $(1989,1997)$, the level of perceived self-motion will rise, peak, and then decline when either the SF or the scene velocity increases. It was, therefore, hypothesized that the level of cybersickness would also rise, peak, and decline as SV is increased by either increasing scene velocity (V) or scene complexity (SF). The hypothesis that the level of cybersickness will decline at high scene velocity is consistent with the limits on pursuit eye movements in fast moving visual scenes (e.g., Cohen, Matsu and Raphan, 1977). Cohen suggests that as eye movement ceased to follow a fast moving visual scene, the level of vection would decline. In addition, this hypothesis is also consistent with the theory of the two-visual system, which states that the ambient visual mode responsible for spatial localization and orientation is not sensitive to luminance variations at high spatial 
frequencies (Held, 1970, Leibowitz and Dichgans, 1980).

\subsection{Brief details of the previously reported Experiment 1 conducted to study the effects of scene velocity}

Details and initial results of the original experiment have previously been reported in So et al. (2000a). Thirty-six healthy Chinese males participated in the experiment. They were exposed to the VE for 30-minute. A duration of 20 to 30 minutes has been commonly used in the studies of cybersickness (e.g., Draper, 1998; Finch and Howarth, 1996; Lampton and Kolasinski et al., 1994; Kolasinski, 1995; Regan, 1995; Stanney and Kennedy, 1997). Three different navigation velocity conditions were used, and each condition had 12 participants. The same VE was used in the three conditions (i.e., same average SFs across conditions) and the differences in SVs were due solely to the changes in navigation velocities.

Using the combined method, the SVs in the fore-and-aft direction for the three conditions were: $0.8,1.1$, and 2.3 cycles $\mathrm{x}$ metres / seconds $\mathrm{x}$ degrees. Table 1 summarizes the measured SVs in the three conditions. In all conditions, participants moved through the VE passively according to a predetermined path. The viewpoint was head-steered and participants were asked to turn their heads from side-to-side (rotating about the vertical axis) periodically and to describe what they observed. This was done so that the participants would be more involved with the VE. The VE, presented on a $48^{\circ} \times 36^{\circ} \mathrm{VR} 4$ head-mounted display, was generated using dVISE software running on a Silicon Graphics Onyx II station. A Polhemus head tracker was used to maintain the correct orientation of the scene with respect to the head. The average frame rate was 30 frames per second and the head movement response lag was about $60 \mathrm{~ms}$. Nausea ratings and SSQ scores were recorded. The nausea ratings were taken verbally at 5 minute intervals using a 7-point Likert scale used in previous work on cybersickness (So, 1994; Lo and So, 2000) and on motion 
sickness (Woodman and Griffin, 1997; Golding and Kerguelen, 1992).

[Insert Table 1 about here]

\subsection{Brief details of the previously reported Experiment 2 conducted to study the effects of scene complexity}

Details and the initial results of the original experiment have previously been reported in So et al. (2000a). There were three conditions: travelling through a VE with either a low, medium or high levels of scene complexity. Using the combined method of calculating SF, the low, medium, and high scene complexity levels corresponded to average radial spatial frequencies of $0.01,0.07$, and $0.24 \mathrm{cpd}$, respectively. At the time of the experiments, the proposed SV metric had not yet been developed and the three complexity conditions were selected by visual inspection. Examples of snap shots taken from the three conditions are shown in Figure 3. The VEs making up the three complexity conditions contained similar polygons and the adjustment in complexity level was mainly achieved by changing the texture of the mappings. The scene velocities were the same in the three conditions and participants were exposed to a 30-minute guided tour of a virtual city. As in Experiment 1, participants made periodic head turns around the yaw axis. Each condition had 12 participants and a total of 36 participants were used. Table 2 summaries the spatial frequencies, scene velocities, and the spatial velocities of the three conditions. The apparatus used was the same as that used in Experiment 1. The average frame rates and response lags of the three VEs had been carefully matched. The average frame rate was 30 frames per second and the head movement response lag was about $60 \mathrm{~ms}$.

[Insert Figure 3 and Table 2 about here] 


\subsection{Results of the re-analyzed data}

The nausea ratings and the increases in SSQ total sickness scores (post-exposure scores - pre-exposure scores) recorded at the end of the 30-minute VR simulations are shown in Figures 4 and 5 as functions of SVs in the fore-and-aft and yaw directions, respectively. The SVs calculated by the three methods: mean, mode, and combined, are shown for comparison. Each data point represents the mean of data from 12 participants. Only SVs in these two axes are shown because the predetermined navigation path did not have much energy in the other axes.

[Insert Figures 4 and 5 about here]

\subsubsection{Comparing the three methods used to calculate SVs} Inspection of Tables 1 and 2 shows that SVs calculated using the mode and the combined methods are similar, while the SVs calculated by the mean method are higher. A possible reason is that most snap shots are dominated by graphics with low SF so that the SFPSD distributions are skewed. An inspection of Figure 3 indicates that both nausea ratings and SSQ total scores increased with increasing SV as calculated by all three methods and do not offer enough evidence to dismiss any method of calculating SVs.

The theory of the two visual systems was again applied to determine which method is most suitable for calculating SVs. Leibowitz et al. (1979) have reported that the level of vection generated from viewing a moving scene is not affected by the introduction of a diopter lens. They concluded that the visual system that is responsible for spatial orientation and vection is not sensitive to high frequency changes in luminance variation (i.e., patterns with high spatial frequencies) - a finding that is consistent with the theory of the two visual systems. A series of 
simulations was conducted to examine the values of SVs after introducing some blurring effects into the 3600 sampled snap shots. This blurring effect was to simulate the visual effects of a diopter lens. The hypothesis was that if the level of vection is not affected by blurring the fine details of the moving scene, then an appropriate measure of SV should also be independent of the blurring effects. The blurring effect was introduced using a photo-editing software (Printshop-Pro). Different levels of blur were available by selecting the 'radius of blur' (the larger the radius, the more severe the blurring effect). Examples of a snap shot before and after applying the blurring effects with a radius of 0, 1, 2 and 4 are shown in Figure 6 (radius of 0 means no blurring).

Results of the simulations indicated that when blurring is introduced, SVs calculated using the mean method reduced significantly $(p<0.001)$ while there was no significant change in SVs calculated by the mode and combined methods ( $p>0.15)$. Consequently, the simulation results suggest that the mode and combined methods are more appropriate than the mean method. The rest of the analyses will focus on data associated with SVs calculated by the mode and combined methods.

Although the effects of blurring did not have a significant effect on the SFs (hence, SVs) calculated by the mode and combined methods, an inspection of Figure 6 indicates that the mode and combined SFs were reduced slightly by the introduction of blurring. This suggests that the level of vection (hence, cybersickness) may not be related to the level of SVs as closely as expected. Future studies to investigate the effects of blurring on cybersickness are desirable (e.g., repeating Leibowitz et al., 1979 study in a VE).

[Insert Figure 6 about here] 


\subsubsection{Effects of SVs in the fore-and-aft direction}

As shown in Figure 4, as SVs increased in the fore-and-aft axis, both the nausea rating (NR) and the total sickness score (TS) increased. Results of ANOVA tests showed that the increases were significant $(p<0.001)$. The hypothesis predicts that as SV increases, the level of cybersickness will increase, peak, and then decline. In this two experiments, both nausea ratings and SSQ scores continued to increase with increasing SVs. A possible reason is that the range of SVs used in Experiment 1 was not high enough so that the level of cybersickness was still in the increasing range. In the previous study by $\mathrm{Hu}$ et al. (1997), the SVs used ranged from $1 \mathrm{cps}$ to $16 \mathrm{cps}$ in the yaw direction (scene with SFs from $1 / 60 \mathrm{cpd}$ to $16 / 60 \mathrm{cpd}$ rotating at $60 \%$ ) and the sickness peaked at $4 \mathrm{cps}$. In this study, the maximum SVs in the yaw axis calculated by the mode and combined methods were 3.1 , and $3.6 \mathrm{cps}$, respectively.

Obviously, there is much difference between the scene presented by Hu et al. (1997) and the two experiments documented by So et al. (2000a). The former consisted of alternating vertical black-and-white stripes on the inside of an optokinetic drum rotating around the yaw axis, while the latter consisted of colored VEs moving mainly in the fore-and-aft and yaw directions. Acknowledging that there are many factors that will affect this comparison (e.g., context of the scene, axis of scene movement), nonetheless, we argue that the possibility of quantifying the scene movement of these two studies (So et al., 2000a and Hu et al., 1997) using the same metric is a vivid demonstration of the benefits of the SV metric.

Correlation tests between SVs and nausea ratings and between SVs and SSQ total scores were conducted. Results indicated that SVs in the fore-and-aft direction were significantly correlated with both nausea ratings and SSQ total scores $(p<0.001)$. Regression models of mean nausea ratings and SSQ total scores as a linear function of SVs are shown in Figure 4. An $R^{2}$ of $>0.97$ and $>0.85$ were obtained between combined SVs and mean nausea ratings and between combined 
SVs and mean SSQ scores, respectively.

\subsubsection{Effects of SVs in the yaw direction}

Inspection of Figures 4 and 5 indicates that the regression analyses with SVs in the yaw direction reported lower values of $R^{2}(>0.61$ and $>0.78)$ than those with SVs in the fore-and-aft direction $(>0.97$ and $>0.85)$. Further investigations indicated that the SVs in the yaw direction had included scene movements introduced by the head turns of the participants. During the study, participants were asked to turn their heads periodically to the left or right to observe some objects in the VE. This resulted in additional scene movement in the yaw direction. According to the sensory conflict theory, these additional scene movements were associated with appropriate physical head movements and, hence, should not cause a conflict between the vestibular cue and visual cue. As a result, SVs in the yaw axis have been recalculated to exclude the additional scene movements introduced by the head movements. This was possible because the head positions and orientations were measured during the studies. The results are shown in Tables 1 and 2 in brackets. Figure 7 illustrates the nausea ratings and SSQ total scores as a function of the revised SVs in the yaw direction. Regression analyses indicated that both the nausea ratings and SSQ total scores increased linearly with revised SVs in the yaw direction $\left(R^{2}=0.99\right.$ and $>0.88$, respectively, Figure 7$)$. Similar to the effects of SVs in the fore-and-aft direction, this result only partially agrees with the hypothesis which predicts that both nausea ratings and SSQ total scores will increase, peak, and then decline as SVs increase. A possible reason has been discussed in Section 3.4.2. After removing the scene movement introduced by the yaw axis head movements, the maximum SV in the yaw axis was reduced to $2.3 \mathrm{cpd}$ (using the combined method). This further supports the argument that the values of SV studied were within the range in which cybersickness would increase with increasing SVs.

The re-analyzed results indicated that increasing SV by increasing either the 
scene velocity (first experiment) or the scene complexity (second experiment) leads to significant increases in both nausea rating and SSQ total scores $(p<0.001$, ANOVAs). Because scene velocity and complexity are the two key components of the proposed SV metric, this suggests that SV can be an appropriate measure to quantify visual scene stimuli for the study of cybersickness. It is acknowledged that this is only the beginning and that there are still many research questions to be answered. Nonetheless, the proposed SV metric should advance the continuous quest to quantify the visual stimuli in the study of cybersickness.

[Insert Figure 7 about here]

\section{DISCUSSION: BENFITS OF THE 'SPATIAL VELOCITY' METRIC}

With the SV metric, it is now possible to quantify the visual stimuli inside a VE, and experiments can be conducted to establish the relationship between the level of visual stimuli (as measured by SV) and the level of cybersickness. Since cybersickness is a type of visually induced motion sickness (Hettinger and Riccio, 1992), studying the relationship between visual stimuli and cybersickness may yield important knowledge about the cause of cybersickness. In addition, the availability of an objective method to measure and quantify the visual stimuli during a VE simulation represents a large step forward toward meaningful comparison among cybersickness studies using different VEs.

At present, calculation of the SV metric is implemented as a MATLab batch file. The authors intend to make this routine available on the Internet so that researchers from all over the world can upload snap shots of their VEs and obtain the average SF_horiz, SF_vert, and SF_radial through their email. It is hoped that this will spur on the quest for the cause of cybersickness as well as the continuous 
development and refinement of the proposed SV metric.

With the ability to meaningfully compare different cybersickness studies, it may be possible to develop a dose value formula to predict the severity of cybersickness. A review of the literature on the motion (sea) sickness dose value (MSDV) indicates that at the center of the MSDV there is a basic unit - ship acceleration along the vertical axis. This acceleration quantifies the heave which is mainly responsible for the generation of seasickness (e.g., Griffin, 1990, British Standards Institution, 1987). The MSDV has the following form (Griffin, 1990):

$$
\operatorname{MSDV}=\left[\int_{0}^{\top} \text { (frequency-weighted ship acceleration along the vertical axis) }^{2} d t\right]^{1 / 2}
$$

where $\mathrm{T}$ is the duration of a voyage

Likewise, the SV metric, which quantifies scene movement that is contributing to the generation of cybersickness, could be used as the basic unit for a cybersickness dose value (CSDV). For example, a simple CSDV could consist of an integration of the SV measurements over the duration of exposure $(T)$ :

$$
\int_{0}^{T} S V d t
$$

In MSDV, the ship acceleration is frequency-weighted. With the proposed CSDV, the hypothesis that cybersickness will increase, peak, and then decline as SVs increase also calls for some sort of weighting function so that SV can be used as a better predictor variable for cybersickness. Future experiments to determine the need and characteristics of the possible frequency weighting function(s) would be beneficial.

It is acknowledged that besides the SV and the duration of exposure, other factors also contribute to the level of cybersickness. For example, subject-related factors (e.g., Cobb et al., 1999; Kolasinski,1995); types and styles of control (e.g., 
Howarth and Finch, 1999; Stanney and Hash, 1998); display parameters (e.g., Dizio and Lackner, 1997; Mon-Williams and Wann, 1998) and time delay (e.g., Dizio and Lackner, 1997; Regan, 1995; So, 1994). In 1992, Kennedy and his colleagues used regression analyses to formulate a dose value to predict the level of blood alcohol level based on a number of different measurements (Kennedy, Turnage and Dunlap, 1992). Similar methodologies can be applied in future studies to extend the proposed CSDV to include the effects of other contributing factors.

It should be noted that, similar to seasickness, there exists a large individual variation in the occurrence and severity level of cybersickness (e.g., seasickness: Griffin, 1990; cybersickness: Lo and So, 2000; Kolasinski, 1995). It is not easy to predict the severity of cybersickness for an individual. Even the MSDV does not predict the severity of sea-sickness for an individual. Rather, it predicts the percentage of passengers who will feel sick after a particular voyage of known duration and ship motion acceleration (Griffin, 1990; British Standards Institution, 1987). While individual predictions of cybersickness cannot be ruled out - especially there have been some promising studies (e.g., Kolasinski and Gilson, 1999) - the primary future of the SV metric is in formulating a cybersickness dose value (CSDV) to predict the percentage of users who will report a certain level of SSQ score after exposure to a VE with known SV values and for a known duration. When this is available, the CSDV can then be combined with the complementary research on individual effects by Kolasinski and others.

\section{CONCLUSIONS, LIMITATIONS and FUTURE RESEARCH}

A metric called spatial velocity (SV) has been developed for quantifying visual scene movement in a VE.

A review of the literature indicates that this SV metric is the first objective metric developed to quantify the visual scene movement in a VE. With such a metric, 
future experiments could be conducted to establish a generic relationship between visual scene movement in a VE and the rated level of cybersickness. The SV metric consists of two components: (i) spatial frequency, which quantifies the spatial variation of luminance information in a VE, and (ii) the r.m.s. navigation velocities of the viewers through the VE. The literature indicates that the SV metric is consistent with the theory of two visual systems, as well as studies on vection perception in the presence of optical flow and studies with optokinetic drums.

The fast Fourier transform has been used to calculate SVs and the methods to determine the dominant spatial frequency across a row or column of a picture have been selected based on studies related to the two visual systems theory.

Data from two previously reported experiments were re-analyzed to determine the relationship between SV and the level of cybersickness. The results showed that both the level of nausea and SSQ total scores increased with increasing SV. Significant correlations were found between SVs and nausea ratings as well as between SVs and SSQ total scores. This verifies that the spatial velocity (SV) measure is a significant predictor of cybersickness and can, therefore, be a suitable quantifying unit for visual scene movement in a virtual environment.

The potential use of this SV unit to establish a cybersickness dose value (CSDV) has also been presented.

Despite the successful introduction of an objective quantifying metric for virtual scene movement, the research is limited in several respects. First, the two previously reported experiments used only male participants. Studies to include female participants are desirable. Second, the range of SVs verified was limited. It has been hypothesized that as SV increases, the level of cybersickness will increase, peak, and then decline. Yet, data from the two reported experiments verified only that as SV increased from 0 to 2.3 metres $x$ cycles per degree $x$ second in the forand-aft direction (using the combined method of calculation), both nausea ratings and SSQ total severity scores increased. Future research should extend the range 
of SVs investigated.

In this study, two snap shots per second were sampled during the navigation tour in the VE in order to calculate the SVs. Further simulations are desirable to determine the optimal number of sample snap shots. In addition, it would be of academic interests to isolate the contributions of the SVs in different axes.

It is planned to post the SV calculation program on the Internet so that other researchers in the field can use it to calculate SVs for their VR simulations. An initial grant for setting up this web site has been obtained, and an announcement will be made when the site is ready.

\section{ACKNOWLEDGEMENTS}

This study was supported by the Hong Kong Research Grants Council through Earmarked Research Grants HKUST738/96E and HKUST6076/99E. The authors are grateful to the reviewers for their constructive comments on the manuscript. A small portion of the study has been presented at the $43^{\text {rd }}$ Annual Meeting of the Human Factors and Ergonomics Society.

\section{REFERENCES}

Bayarri, S., Fernandez, M. and Perz, M. (1996) Virtual reality for a driving simulation. J. of Commun. ACM (USA), Vol.39, No.5, pp.72-6.

Bendat, J.S. and Piersol, A.G. (1986) Random data: analysis and measurement procedures. New York: Wiley, $2^{\text {nd }}$ Edition, ISBN 0-4710-4000-2, pp. 300-310.

Benson, A.J. (1984) Motion sickness. In: M.R. Dix and J.S. Hood (Eds.). Vertigo, New York: Wiley, Chapter 19, pp. 391-426. 
Boff, K.R. and Lincoln, J.E. (1988) Engineering data compendium: Human Perception and Performance, AAMRL, Wright-Patterson AFB, Ohio, USA.

Brandt T.H. (1973) Differential effects of central versus peripheral vision on egocentric and exocentric motion perception, Exp. Brain Res, 16, pp. 476-491.

British Standards Institution (1987) Measurement and evaluation of human exposure to whole-body mechanical vibration and repeated shock: BS6841. British Standards Institution, London.

Cobb, S.V.G., Nichols, S., Ramsey, A. and Wilson, J. (1999) Virtual reality induced symptoms and effects (VRISE). Presence, 8, pp. 169-186.

Cohen, B., Matsuo, V. and Raphan, T. (1977) Quantitative analysis of the locity characteristics of optokinetic after-nystagmus. J. Physiol., 270, pp. 321-344.

Dichgans, J. and Brandt, T.H. (1978) Visual-vestibular interactions: effects on selfmotion perception and postural control. In: R. Held, H. Leibowitz, H.L. Teuber (Eds.). Handbook of Sensory Physiology (vol.8). New York: Springer-Verlag, pp. 755-804.

Dizio, P. and Lackner, J.R. (1991) Motion sickness susceptibility in parabolic flight and velocity storage activity. Aviation, Space, and Environmental Medicine, 62, pp. 300-307.

DiZio, P. and Lackner, J.R. (1997) Circumventing side effects of immersive virtual environments. Proceeding of the $7^{\text {th }}$ International Conference on Human-Computer Interaction, 24-29 August, San Francisco, CA. 
Draper, M.H. (1998) The effects of image scale factor on vestibulo-ocular reflex adaption and simulator sickness in head-coupled virtual environments. Proceedings of the Human Factors and Ergonomics Society $42^{\text {nd }}$ Annual Meeting, 5-9 September, Chicago, USA, pp. 1481-1485.

Ehrlich, J.A (1997) Simulator sickness and HMD configuration. Proceedings of the SPIE Conference on Telemanipulator \& Telepresence Technology, SPIE Vol. 3206, pp. $170-178$.

Ehrlich, J.A., Singer, M.J. and Allen, R.C. (1998) Relationships between headshoulder divergences and sickness in a virtual environment. Proceedings of the Human Factors and Ergonomics Society $42^{\text {nd }}$ Annual Meeting, 5-9 September, Chicago, USA, pp. 1471-1475.

Gibson, J.J. (1947) Motion picture testing and research. Army Air Force Aviation Psychology Research Report, No.7, (NTIS No. AD-651 783), Government Printing Office, Washington, DC.

Gibson, J.J. (1950) The perception of the visual world. Boston: Houghton Mifflin.

Gibson, J.J., Olum, P. and Rosenblatt, F. (1955) Parallax and perspective during aircraft landings. American Journal of Psychology, 68, pp. 372-385.

Golding, J.F. and Kerguelen, M. (1992) A comparison of the nauseogenic potential of low-frequency vertical versus horizontal linear oscillation. Aviation, Space, and Environmental Medicine, 63, pp. 491-497. 
Griffin, M.J. (1990) Handbook of human vibration. Academic Press, ISBN 0-12303040-4, pp. 271-330.

Held, R. (1970) Two modes of processing spatially distributed visual stimulation. In: F.O. Schmitt (Ed.). The Nurosciences: Second Study Program. New York: Rockefeller University Press.

Henn, V., Cohen, B. and Young, L.R. (1980) Visual-vestibular interaction motion perception and the generation of nystagmus. Neuro. Res. Prog. Bull, 18, pp. 459651.

Hettinger, L.J., Owen, D.H. and Warren, R. (1985) Global optical flow pattern information for loss in altitude. In: D.H. Owen (Ed.). Optical and event-duration variables affecting self-motion perception (Interim Tech. Rep. For AFHRL Contact No. F33615-83-K-0038). Ohio State University, Department of Psychology, Aviation Psychology Laboratory.

Hettinger, L.J., Berbaum, K.S., Kennedy, R.S., Dunlap, W.P. and Nolan, M.D. (1990) Vection and simulator sickness. Military Psychology, 2, pp.171-181.

Hettinger, L.J. and Riccio, G.E. (1992) Visually induced motion sickness in virtual environments. Presence: Teleoperators and Virtual Environments, 1, pp. 306-310.

Howarth, P.A. and Costello, P.J. (1997) The occurrence of virtual simulation sickness symptoms when an HMD was used as a personal viewing system. Display, vol.18, no.2, pp. 107-116.

Howarth, P.A. and Finch, M. (1999) The nauseogenicity of two methods of navigating 
within a virtual environment. Applied Ergonomics, 30, pp. 39-45.

Howarth, P.A. and Hill, K.J. (1999) The maintenance of habituation to virtual simulation sickness. Proceedings of $\mathrm{HCl}$ International '99, 22-26 August, Munich, Germany, pp. 137-141.

Hu, S., Stern, R.M., Vasey, M.W. and Koch, K.L. (1989) Motion sickness and gastric myelectric activity as a function of speed of rotation of a circular vection drum. Aviation, Space, and Environmental Medicine, 60, pp. 411-414.

Hu, S., Davis, M.S., Klose, A.H., Zabinsky, E.M., Meux, S.P., Jacobson, H.A., Westfall, J.M. and Gruber, M.B. (1997) Effects of spatial frequency of a vertically striped rotating drum on vection-induced motion sickness. Aviation, Space, and Environmental Medicine. Vol.68, No.4, April, pp.306-311.

Kennedy, R.S. and Frank, L.H. (1984) A review of motion sickness with special reference to simulator sickness. (NAVTRAEQUIP-CEN 81-C-0105-16) Naval Training Equipment Center. (Technical Report, Transportation Research Board, National Research Council), Orlando, USA.

Kennedy, R.S. and Stanney, K.M. (1997) Aftereffects of virtual environment exposure: psychometric issues. Proceeding of the $7^{\text {th }}$ International Conference on Human-Computer Interaction, 24-29 August, San Francisco, CA.

Kennedy, R.S., Berbaum, K.S., Dunlap, W.P. and Hettinger, L.J. (1996) Developing automated methods to qualify the visual stimulus for cybersickness. Proceedings of the $40^{\text {th }}$ Human Factors and Ergonomics Society's Annual Meeting, pp. 1126-1130. 
Kennedy, R.S., Berbaum, K.S. and Smith, M.G. (1993) Methods for correlating visual scene elements with simulator sickness incidence. Proceedings of the Human Factors and Ergonomics Socity 37th Annual Conference, pp.1252-1256.

Kennedy, R. S., Hettinger, L. J., Harm, D. L., Ordy, J. M., and Dunlap, W. P. (1996)

Psychophysical scaling of circular vection (CV) produced by optokinetic (OKN) motion: Individual differences and effects of practice. Journal of Vestibular Research, 6, pp. 331-341.

Kennedy, R.S., Lane, N.E., Berbaum, K.S. and Lilienthal, M.G. (1993) Simulator Sickness Questionnaire (SSQ): a new method for quantifying simulator sickness. International Journal of Aviation Psychology, 3(3), pp. 203-320.

Kennedy, R.S., Lanham, S., Drexler, J.M., Massey, C.J. and Lilienthal, M.G. (1995) Cybersickness in several flight simulators and VR devices: a comparison of incidences, symptom profiles, measurement techniques and suggestions for research. Proceedings of the Conference of the FIVE Working Group. London, 1819, December, 1995. pp. 243-251.

Kennedy, R.S., Turnage, J.J. and Dunlap, W.P. (1992) The use of dose equivalency as a risk assessment index in behavioral neurotoxicology. Neurotoxicology and Teratology, 14, pp. 167-175.

Koch, K.L., Stern, R.M., Vasey, M.W., Seaton, J.F., Demers, L.M. and Harrison, T.S. (1990) Neuroendocrine and gastric myoelectrical responses to illusory self-motion in humans. American Journal of Physiology, 258, pp. 304-310.

Kolasinski, E.M. (1995) Simulator sickness in virtual environments. United States 
Army Research Institute for the Behavioral and Social Sciences. Technical Report 1027.

Kolasinski, E.M. and Gilson, R.D. (1999) An investigation into the predictive modeling of VE sickness. Proceedings of $\mathrm{HCl}$ International '99, 22-26 August, Munich, Germany, pp. 142-146.

Lampton, D.R., Kolasinski, E.M., Knerr, B.W., Bliss, J.P., Bailey, J.H. and Witmer, B.G. (1994) Side effects and aftereffects of immersion in virtual environments. Proceedings of the Human Factors and Ergonomics Society $38^{\text {th }}$ Annual Meeting. Santa Monica, CA, pp. 1154-1157.

Leibowitz, H.W. and Dichgans, J. (1980) The ambient visual system and spatial orientation. Proceedings of AGARD Conference on spatial disorientation in flight: current problems, Bodo, Norway, (AGARD-CP-287), pp. B4-1 to B4-4.

Leibowitz, H.W. and Post, R.B. (1982) The two modes of processing concepts and some implications. In: J. Beck (Ed.) Organization and Representation in Perception, Hillsdale, HJ: Lawerence Erlbaum Associates, pp. 343 - 363.

Leibowitz, H.W., Shupert-Rodemer, C., and Dichgans, J. (1979) The independence of dynamic spatial orientation from luminance and refractive error. Perception and Psychophysics, 25, pp. 75-79.

Livingstone, M. and Hubel, D. (1988) Segregation of form, color, movement, and depth: anatomy, physiology, and perception. Science, 240, pp. 740-749.

Lo, W.T. and So, R.H.Y. (2000) Cybersickness in the presence of scene rotational 
movements in different axes. Submitted to Applied Ergonomics.

McCauley, M.E. and Sharkey, T.J. (1992) Cybersickness: perception of self-motion in virtual environments. Presence, 1(3), pp. 311-318.

Mon-Williams, M. and Wann J. (1998) Binocular virtual reality displays: when problem do and don't occur. Proceedings of the Human Factors and Ergonomics Society $42^{\text {nd }}$ Annual Meeting, 5-9, October, Chicago, USA, pp. 42-48.

Mon-Williams, M., Plooy, A., Burgess-Limerick, R. and Wann J. (1998) Gaze angle: a possible mechanism of visual stress in virtual reality headsets. Ergonomics, 41, pp. 280-285.

Muller, C.H., Wiest, G. and Deecks, L. (1990) Vertically moving visual stimuli and vertical vection - a tool against space motion sickness? Proceedings of the Fourth European Symposium on Life Sciences Research in Space, 28 May to 1 June, Trieste, Italy, (ESA SP-307).

Oman, C.M. (1982) A heuristic mathematical model for the dynamics of sensory conflict and motion sickness. Acta Oto-laryngologica Supplement 392.

Oman, C.M. (1993) Sensory conflict in motion sickness: an observer theory approach. In: S.R. Ellie (Ed.). Pictorial communication in virtual and real environments. Taylor \& Francis, pp. 362 - 375. 
Oppenheim, A.V., Willsky, A.S. and Nawab, H. (1997) Signals and systems.

Upper Saddle River, N.J. : Prentice Hall.

Owen, D.H. and Freeman, S.J. (1986) Effects of preview duration, optical flow rate, and optical texture density on sensitivity to loss in altitude. In: D.H. Owen (Ed.), Perception and control of simulated self-motion. Interim Technical Report for AFHRL Contract No. F33615-83-K-0038, The Ohio State University, Department of Psychology, Aviation Psychology Laboratory.

Owen, D.H. and Jensen, R.S. (1981) Methodological approaches to identifying relevant features for visual flight simulation. Final Report to the Air Force Office of Scientific Research Directorate of Life Science, Bolling Air Force Base, on Contract F49620-79-0070. Aviation Psychology Laboratory, Ohio State University, Columbus, Ohio.

Owen, D.H. and Warren, R. (1982) Optical variables as measures of performance during simulated flight. Proceedings of the $26^{\text {th }}$ Human Factors Society Annual Meeting, pp. $312-315$.

Owen, D.H. and Warren, R. (1987) Perception and control of self-motion: implications for visual simulation of vehicular locomotion. In: L.S. Mark, J.S. Warm and R.L. Huston (Eds.) Ergonomics and Human Factors: Recent Research, NY: Springer-Verlag, pp. 40-69.

Owen, D.H., Warren, R., Jensen, R.S., Mangold, S.J. and Hettinger, L.J. (1981) Optical information for detecting loss in one's own forward speed. Acta Psychologica, 48, pp. 203-213. 
Owen, D.H., Wolpert, L. and Warren, R. (1984) Effects of optical flow acceleration, edge acceleration, and viewing time on the perception of egospeed acceleration. In: D.H. Owen (Ed.) Optical flow and texture variables useful in detecting decelerating and accelerating self-motion (AFHRL-TP-84-4, AD-A148-718). Operations Training Division, Air Force Human Resources Laboratory, Williams AFB, Arizona, USA.

Owen, D.H., Hettinger, L.J., Pallos, I.E. and Fogt, J.C. (1985) The influence of preview period and global optical flow rate on sensitivity to decelerating self motion. In: D.H. Owen (Ed.) Optical and event-duration variables affecting self-motion perception (Interim Technical Report for AFHRL Contract No. F33615-83-K-0038) Ohio State University, Department of Psychology, Aviation Psychology Laboratory.

Previc, F.H., Varner, D.C. and Gillingham, K.K (1992) Visual scene effects on the somatogravic illusion. Aviation, Space, and Environmental Medicine, December, pp. $1060-1063$.

Prothero, J.D., Draper, M.H., Furness, T.A., Parker, D.E. and Wells, M.J. (1999) The use of an independent visual background to reduce simulator side-effects. Aviation, Space, and Environmental Medicine, 70, pp. 277-283.

Reason, J.T. (1978) Motion sickness adaptation: a neural mismatch model. Journal of the Royal Society of Medicine, 71, pp. 819-829.

Reason, J.T and Brand, J.J. (1975) Motion Sickness. Academic Press.

Regan, C (1995) An investigation into nausea and other side-effects of headcoupled immersive virtual reality. Virtual Reality (1995) Vol.1, No.1, pp. 17-32. 
Schalkoff, R.J. (1989) Digital image processing and computer vision. New York: Wiley.

Schneider, G.E. (1967) Contrasting visuomotor functions of tectum and cortex in the golden hamster. Psychologische Forschung, 31, pp. 52-62.

Slater, M., Linakis, V., Usoh, M. and Kooper, R. (1996) Immersion, presence and performance in virtual environments: an experiment with tri-dimensional chess. Proceedings of the ACM Symposium on Virtual Reality Software and Technology, 14 July, pp. 163-172.

So, R.H.Y. (1994). An investigation of the effects of lags on motion sickness with a head-coupled visual display. Proceedings of the United Kingdom Informal Group Meeting on Human Response to Vibration held at the Institute of Naval Medicine, Alverstoke, Gosport, Hants, 19th to 21st September 1994.

So, R.H.Y., Lo, W.T. and Ho, A.T.K. (2000a) Scene movement: an important cause of cybersickness. In: M. Mahtun, M. Helander (Eds.). Seminars on Virtual Reality: selected tools and applications. Academic Press: London.

So, R.H.Y., Lo, W.T. and Ho, A.T.K. (2001) Effects of navigation speed on the level of cybersickness caused by an immersive virtual environment. Human Factors, 43, 2001, pp.452-561.

Stanney, K.M. and Hash, P. (1998) Locus of user-initiated control in virtual environments: influences on cybersickness. Presence, 7, pp. 447-459.

Stanney, K.M. and Kennedy, R.S. (1998) Aftereffects from virtual environment 
exposure: how long do they last? Proceedings of the Human Factors and Ergonomics Society $42^{\text {nd }}$ Annual Meeting, 5-9, October, Chicago, USA, pp. 14761480.

Stanney, K.M., Kennedy, R.S., Drexler, J.M. and Harm, D.L. (1999) Motion sickness and proprioceptive aftereffects following virtual environment exposure. Applied Ergonomics, Vol. 30, No. 1, pp. 27-39.

Stanney, K.M., Salvendy, G. and 25 other authors (1998) Aftereffects and sense of presence in virtual environments: formulation of a research and development agenda. International Journal of Human-Computer Interaction, 10, pp. 135-187.

Stern, R.M. and Koch,K.L. (1991) Motion sickness. Motility 1991;16, pp. 11-14.

Stern, R.M., Hu, S., Leblanc, B.S. and Koch, K.L. (1993) Chinese hypersusceptibility to vection-induced motion sickness. Aviation, Space, and Environmental Medicine. Sept. pp. 827 - 832.

Stern, R.M., Hu, S., Vasey, M.W. and Koch, K.L. (1989) Adaptation to vectioninduced symptoms of motion sickness. Aviation, Space, and Environmental Medicine, 60, pp 566-572.

Stern, R.M., Koch,K.L., Leibowitz, H.W., Shupert, C.L. and Stewart, W.R. (1985) Tachygastria and motion sickness. Aviation, Space and Environmental Medicine. 56, pp. 1074-1077.

Tobias, S.B. and Owen, D.H. (1984) Optical flow and texture variables useful in detecting decelerating self-motion. In D.H. Owen (Ed.). Optical flow and texture 
variables useful in detecting decelerating and accelerating self-motion. (AFHRL-TP84-4, AD-A148718), Operations Training Division, Air Force Human Resources Lab, Williams Air Force Base, Arizona, USA.

Wells, M.J., Venturino, M. and Osgood, R.K. (1989) The effects of field-of-view size on performance at a simple simulated air-to-air mission. SPIE Conf. On HelmetMounted Displays, 28-29, March, Orlando, Florida, SPIE Vol.1116, pp.126-137.

Wilson, J.R. (1996) Effects of participating in virtual environments: a review of current knowledge. Safety Science, 23, pp. 39-51.

Wilson, J.R., Nichols, S. and Haldane, C. (1997) Presence and side effects: complementary or contradictory? Proceedings of the 7th International Conference on Human-Computer Interaction, August, San Francisco, pp. 889-892.

Woodman, P.D. and Griffin, M.J. (1997) Effect of Direction of Head Movement on Motion Sickness Caused by Coriolis Stimulation. Aviation, Space, and Environmental Medicine, 68, pp. 93-98. 


\section{FIGURE AND TABLE CAPTIONS}

Table 1. Spatial frequencies (SF), scene velocities (V), and spatial velocities (SV) for the 3 conditions in Experiment 1. (The 3 conditions differ in their scene velocities.) (SFs and SVs calculated by the mean, mode, and combined (comb.) methods are shown).

Table 2. Spatial frequencies (SF), scene velocities (V), and spatial velocities (SV) for the 3 conditions in the Experiment 2. (The 3 conditions differ in their spatial frequencies, i.e., scene complexities) (SFs and SVs calculated by the mean, mode, and combined (comb.) methods are shown).

Figure 1. An illustration of how spatial frequency power spectral density of a row is measured.

Figure 2. Spatial frequencies of two examples: 'A' a black-and-white strip pattern;

' $\mathrm{B}$ ' a sampled scene from a virtual environment (field-of-view: $48^{\circ}$ horizontal $\times 36^{\circ}$ vertical; $640 \times 480$ pixels).

Figure 3. Spatial frequency measurements for sampled snap shots of a VR guided tour with low, medium and high scene complexity levels (field-of-view: $48^{\circ}$ horizontal $\times 36^{\circ}$ vertical, $640 \times 480$ pixels).

Figure 4. Mean nausea ratings and SSQ total scores obtained after a 30-minute VR simulation as functions of spatial velocity (SV) in the fore-and-aft direction calculated using 3 different methods (cycles $x$ meters / degree $x$ second). Data re-analyzed from two experiments previously reported by So et al. (2000a).

Figure 5. Mean nausea ratings and SSQ total scores obtained after a 30-minute VR simulation as functions of spatial velocity (SV) in the yaw direction calculated using 3 different methods (cycles / degree). Data re-analyzed from two experiments previously reported by So et al. (2000a). 
Figure 6. Spatial frequency measurements for a sample snap shot with and without blurring effects. (blurring radius of $0,1,2$, and 4 using the software Printshop-Pro) (field-of-view: $48^{\circ}$ horizontal $\times 36^{\circ}$ vertical, 640 × 480 pixels).

Figure 7. Mean nausea ratings and SSQ total scores obtained after a 30-minute VR simulation as a function of spatial velocity (SV) in the yaw direction calculated using the mode and combined methods (cycles / degree). Scene movements in response to head yaw movements of the participants have been excluded from the calculation of SVs. Data reanalyzed from two experiments previously reported by So et al. (2000a). 
Table 1. Spatial frequencies (SF), scene velocities (V), and spatial velocities (SV) for the 3 conditions in Experiment 1. (The 3 conditions differ in their scene velocities.) (SFs and SVs calculated by the mean, mode, and combined (comb.) methods are shown).

\begin{tabular}{|c|c|c|c|c|}
\hline \multirow{2}{*}{\multicolumn{2}{|c|}{ Medium }} & \multicolumn{3}{|c|}{3 levels of scene velocities } \\
\hline & & Low & Medium & high \\
\hline \multirow{6}{*}{$\begin{array}{l}\text { Scene } \\
\text { Velocity } \\
\text { r.m.s. (V) }\end{array}$} & Fore-\&-aft (x) (metre/s) & 3.3 & 4.3 & 9.5 \\
\hline & Lateral (y) (metre/s) & 1.2 & 1.6 & 3.4 \\
\hline & Vertical (z) (metre/s) & 0.2 & 0.3 & 0.6 \\
\hline & Roll (deg./s) & 0.5 & 0.6 & 1.4 \\
\hline & Yaw (deg./s) & $14.2<3.8>^{*}$ & $16.2<5>^{*}$ & $17.3<11>^{*}$ \\
\hline & Pitch (deg./s) & 0.6 & 0.8 & 1.6 \\
\hline \multirow[t]{3}{*}{$\begin{array}{l}\text { Spatial } \\
\text { frequency } \\
\text { (SF) }\end{array}$} & Horizontal (cycle/deg.) & \multicolumn{3}{|c|}{$\begin{array}{c}0.79 \text { (mean) } \\
0.18 \text { (mode) } \\
0.21 \text { (combined) }\end{array}$} \\
\hline & Vertical (cycle/deg.) & \multicolumn{3}{|c|}{$\begin{array}{c}0.60 \text { (mean) } \\
0.09 \text { (mode) } \\
0.09 \text { (combined) }\end{array}$} \\
\hline & Radial (cycle/deg.) & \multicolumn{3}{|c|}{$\begin{array}{c}1.04 \text { (mean) } \\
0.21 \text { (mode) } \\
0.24 \text { (combined) }\end{array}$} \\
\hline \multirow{6}{*}{$\begin{array}{l}\text { Spatial } \\
\text { velocity } \\
\text { (SV) } \\
\text { using SFs } \\
\text { by 'mean' } \\
\text { Method }\end{array}$} & Fore-\&-aft (x) (mc/ds) & 3.43 & 4.47 & 9.88 \\
\hline & Lateral (y) (mc/ds) & 0.95 & 1.26 & 2.69 \\
\hline & Vertical (z) (mc/ds) & 0.12 & 0.18 & 0.36 \\
\hline & Roll (cycle/s) & 0.52 & 0.62 & 1.46 \\
\hline & Yaw (cycle/s) & $11.22<3>^{*}$ & $12.80<4>^{*}$ & $13.67<8.7>^{*}$ \\
\hline & Pitch (cycle/s) & 0.36 & 0.48 & 0.96 \\
\hline \multirow{6}{*}{$\begin{array}{l}\text { Spatial } \\
\text { velocity } \\
\text { (SV) } \\
\text { using SFs } \\
\text { by 'mode' } \\
\text { method }\end{array}$} & Fore-\&-aft (x) (mc/ds) & 0.69 & 0.90 & 2.00 \\
\hline & Lateral $(\mathrm{y})(\mathrm{mc} / \mathrm{ds})$ & 0.22 & 0.29 & 0.61 \\
\hline & Vertical (z) (mc/ds) & 0.02 & 0.03 & 0.05 \\
\hline & Roll (cycle/s) & 0.11 & 0.13 & 0.29 \\
\hline & Yaw (cycle/s) & $2.56<0.7>^{\star}$ & $2.92<0.9>^{*}$ & $3.11<2>^{*}$ \\
\hline & Pitch (cycle/s) & 0.05 & 0.07 & 0.14 \\
\hline \multirow{6}{*}{$\begin{array}{l}\text { Spatial } \\
\text { velocity } \\
\text { (SV) } \\
\text { using SFs } \\
\text { by 'comb.' } \\
\text { method }\end{array}$} & Fore-\&-aft $(\mathrm{x})(\mathrm{mc} / \mathrm{ds})$ & 0.79 & 1.03 & 2.28 \\
\hline & Lateral $(\mathrm{y})(\mathrm{mc} / \mathrm{ds})$ & 0.25 & 0.34 & 0.71 \\
\hline & Vertical (z) (mc/ds) & 0.02 & 0.03 & 0.05 \\
\hline & Roll (cycle/s) & 0.12 & 0.14 & 0.34 \\
\hline & Yaw (cycle/s) & $2.98<0.8>^{*}$ & $3.40<1.1>^{*}$ & $3.63<2.3>^{*}$ \\
\hline & Pitch (cycle/s) & 0.05 & 0.07 & 0.14 \\
\hline
\end{tabular}

Keys:

mc/ds: metre $x$ cycle / degree $x$ second

* scene movements due to head movements in the yaw axis has been excluded 
Table 2. Spatial frequencies (SF), scene velocities (V), and spatial velocities (SV) for the 3 conditions in the Experiment 2. (The 3 conditions differ in their spatial frequencies, i.e., scene complexities) (SFs and SVs calculated by the mean, mode, and combined (comb.) methods are shown).

\begin{tabular}{|c|c|c|c|c|}
\hline \multirow{2}{*}{\multicolumn{2}{|c|}{ Medium }} & \multicolumn{3}{|c|}{ Levels of scene complexity } \\
\hline & & Low & \begin{tabular}{|l|l} 
medium & \\
\end{tabular} & high \\
\hline \multirow{6}{*}{$\begin{array}{c}\text { Scene } \\
\text { Velocity } \\
\text { r.m.s. (V) }\end{array}$} & Fore-\&-aft (x) (metre/s) & \multicolumn{3}{|c|}{9.5} \\
\hline & Lateral (y) (metre/s) & \multicolumn{3}{|c|}{3.4} \\
\hline & Vertical (z) (metre/s) & \multicolumn{3}{|c|}{0.6} \\
\hline & Roll (deg./s) & \multicolumn{3}{|c|}{1.4} \\
\hline & Yaw (deg./s) & \multicolumn{3}{|c|}{$17.3<11>^{*}$} \\
\hline & Pitch (deg./s) & \multicolumn{3}{|c|}{1.6} \\
\hline \multirow{9}{*}{$\begin{array}{l}\text { Spatial } \\
\text { frequency } \\
\text { (SF) }\end{array}$} & Horizontal (cycle/deg.) & 0.03 (mean) & 0.22 (mean) & 0.79 (mean) \\
\hline & & 0.01 (mode) & 0.06 (mode) & 0.18 (mode) \\
\hline & & 0.01 (comb.) & 0.07 (comb.) & 0.21 (comb.) \\
\hline & Vertical (cycle/deg.) & 0.02 (mean) & 0.08 (mean) & 0.60 (mean) \\
\hline & & 0.00 (mode) & 0.02 (mode) & 0.09 (mode) \\
\hline & & 0.00 (comb.) & 0.02 (comb.) & 0.09 (comb.) \\
\hline & Radial (cycle/deg.) & 0.04 (mean) & 0.24 (mean) & 1.04 (mean) \\
\hline & & 0.01 (mode) & 0.06 (mode) & 0.21 (mode) \\
\hline & & 0.01 (comb.) & 0.07 (comb.) & 0.24 (comb.) \\
\hline \multirow{6}{*}{$\begin{array}{l}\text { Spatial } \\
\text { velocity } \\
\text { (SV) } \\
\text { using SFs } \\
\text { by 'mean' } \\
\text { Method }\end{array}$} & Fore-\&-aft (x) (mc/ds) & 0.38 & 2.28 & 9.88 \\
\hline & Lateral $(\mathrm{y})(\mathrm{mc} / \mathrm{ds})$ & 0.10 & 0.75 & 2.69 \\
\hline & Vertical (z) (mc/ds) & 0.01 & 0.05 & 0.36 \\
\hline & Roll (cycle/s) & 0.06 & 0.34 & 1.46 \\
\hline & Yaw (cycle/s) & $0.52<0.3>^{*}$ & $3.81<2.4>^{*}$ & $13.67<8.7>^{*}$ \\
\hline & Pitch (cycle/s) & 0.03 & 0.13 & 0.96 \\
\hline \multirow{6}{*}{$\begin{array}{l}\text { Spatial } \\
\text { velocity } \\
\text { (SV) } \\
\text { using SFs } \\
\text { by 'mode' } \\
\text { method }\end{array}$} & Fore-\&-aft (x) (mc/ds) & 0.10 & 0.57 & 2.00 \\
\hline & Lateral $(\mathrm{y})(\mathrm{mc} / \mathrm{ds})$ & 0.03 & 0.20 & 0.61 \\
\hline & Vertical (z) (mc/ds) & 0.00 & 0.01 & 0.05 \\
\hline & Roll (cycle/s) & 0.01 & 0.08 & 0.29 \\
\hline & Yaw (cycle/s) & $0.17<0.1>^{*}$ & $1.04<0.7\rangle^{*}$ & $3.11<2.0>^{*}$ \\
\hline & Pitch (cycle/s) & 0.00 & 0.03 & 0.14 \\
\hline \multirow{6}{*}{$\begin{array}{l}\text { Spatial } \\
\text { velocity } \\
\text { (SV) } \\
\text { using SFs } \\
\text { by comb. } \\
\text { methods }\end{array}$} & Fore-\&-aft (x) (mc/ds) & 0.10 & 0.67 & 2.28 \\
\hline & Lateral $(\mathrm{y})(\mathrm{mc} / \mathrm{ds})$ & 0.03 & 0.24 & 0.71 \\
\hline & Vertical (z) (mc/ds) & 0.00 & 0.01 & 0.05 \\
\hline & Roll (cycle/s) & 0.01 & 0.10 & 0.34 \\
\hline & Yaw (cycle/s) & $0.17<0.1>^{*}$ & $1.21<0.8>^{*}$ & $3.63<2.3>^{*}$ \\
\hline & Pitch (cycle/s) & 0.00 & 0.03 & 0.14 \\
\hline
\end{tabular}

Keys:

mc/ds: metre $x$ cycle / degree $x$ second

* scene movements due to head movements in the yaw axis has been excluded 

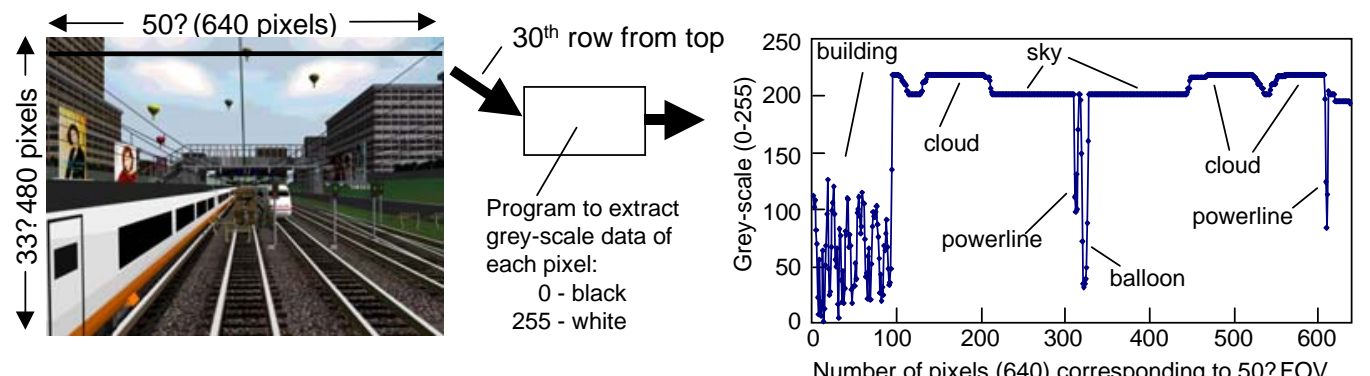

Average SF according to

Mean method

$=0.19 \mathrm{cpd}$

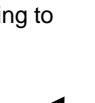

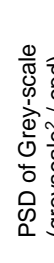
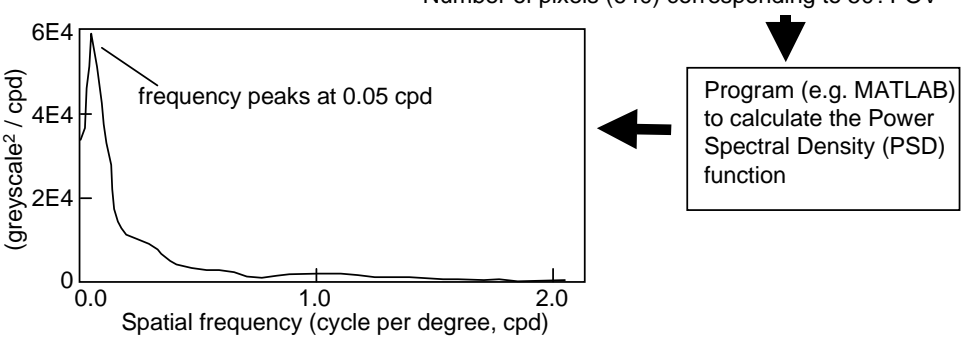

Mode method

$=0.05 \mathrm{cpd}$

Combined method

$=0.05 \mathrm{cpd}$

Figure 1. 
[A]

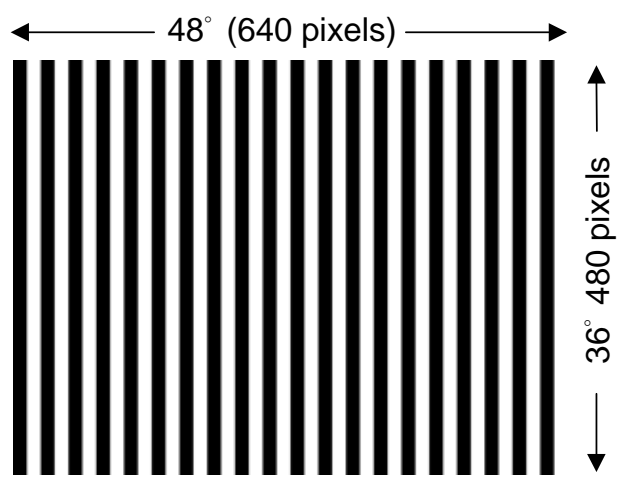

Mean:

Mode:

Combined: $\quad 0.42 \mathrm{cpd}$
SF_vert. $0.00 \mathrm{cpd}$ $0.00 \mathrm{cpd}$ $0.00 \mathrm{cpd}$
[B]

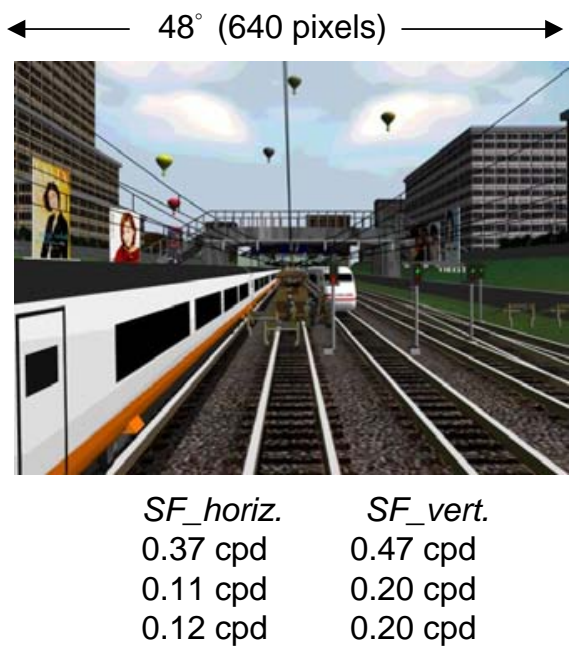

Figure 2. 


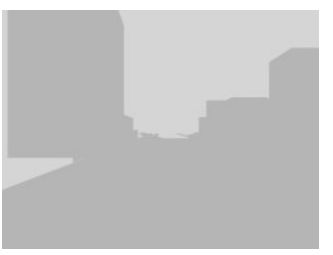

Low scene complexity

SF_horiz. SF_vert. SF_radial $0.07 \mathrm{cpd} \quad 0.07 \mathrm{cpd} \quad 0.10 \mathrm{cpd}$

Mean:

$\begin{array}{llll}\text { Mode: } & 0.02 \mathrm{cpd} & 0.01 \mathrm{cpd} & 0.03 \mathrm{cpd} \\ \text { Combined: } & 0.02 \mathrm{cpd} & 0.02 \mathrm{cpd} & 0.03 \mathrm{cpd}\end{array}$

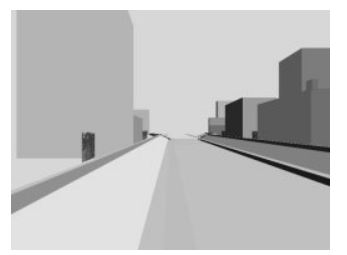

Medium scene complexity

SF_horiz. SF_vert. SF_radial

$0.15 \mathrm{cpd} \quad 0.07 \mathrm{cpd} \quad 0.17 \mathrm{cpd}$

$0.02 \mathrm{cpd} \quad 0.02 \mathrm{cpd} \quad 0.04 \mathrm{cpd}$

$0.02 \mathrm{cpd} \quad 0.02 \mathrm{cpd} \quad 0.04 \mathrm{cpd}$

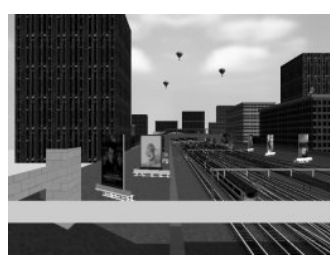

High scene complexity SF_horiz. SF_vert. SF_radial $0.71 \mathrm{cpd} \quad 0.61 \mathrm{cpd} \quad 0.93 \mathrm{cpd}$ $0.05 \mathrm{cpd} \quad 0.16 \mathrm{cpd} \quad 0.17 \mathrm{cpd}$ $0.07 \mathrm{cpd} \quad 0.17 \mathrm{cpd} \quad 0.18 \mathrm{cpd}$

Figure 3. 


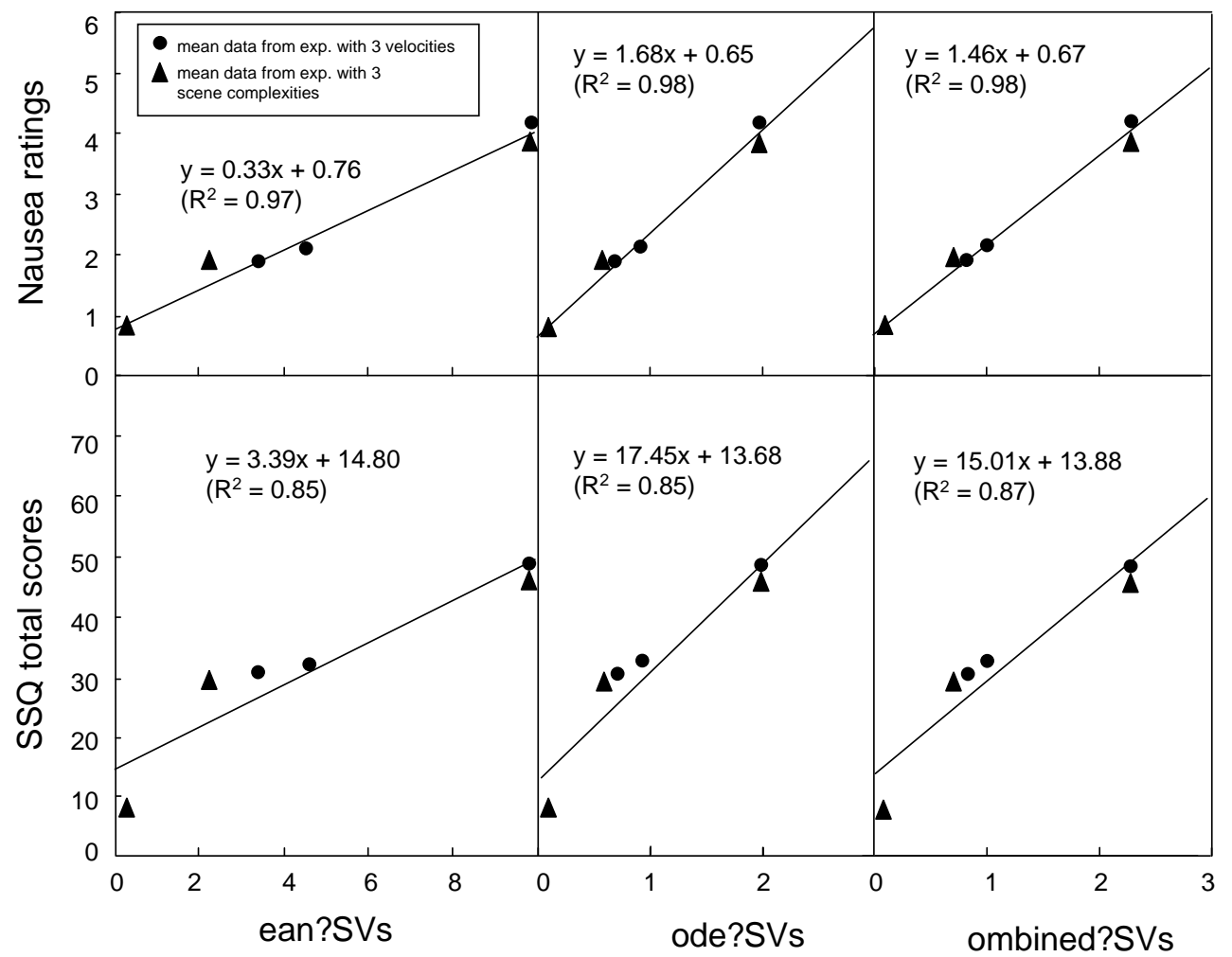

Figure 4. 


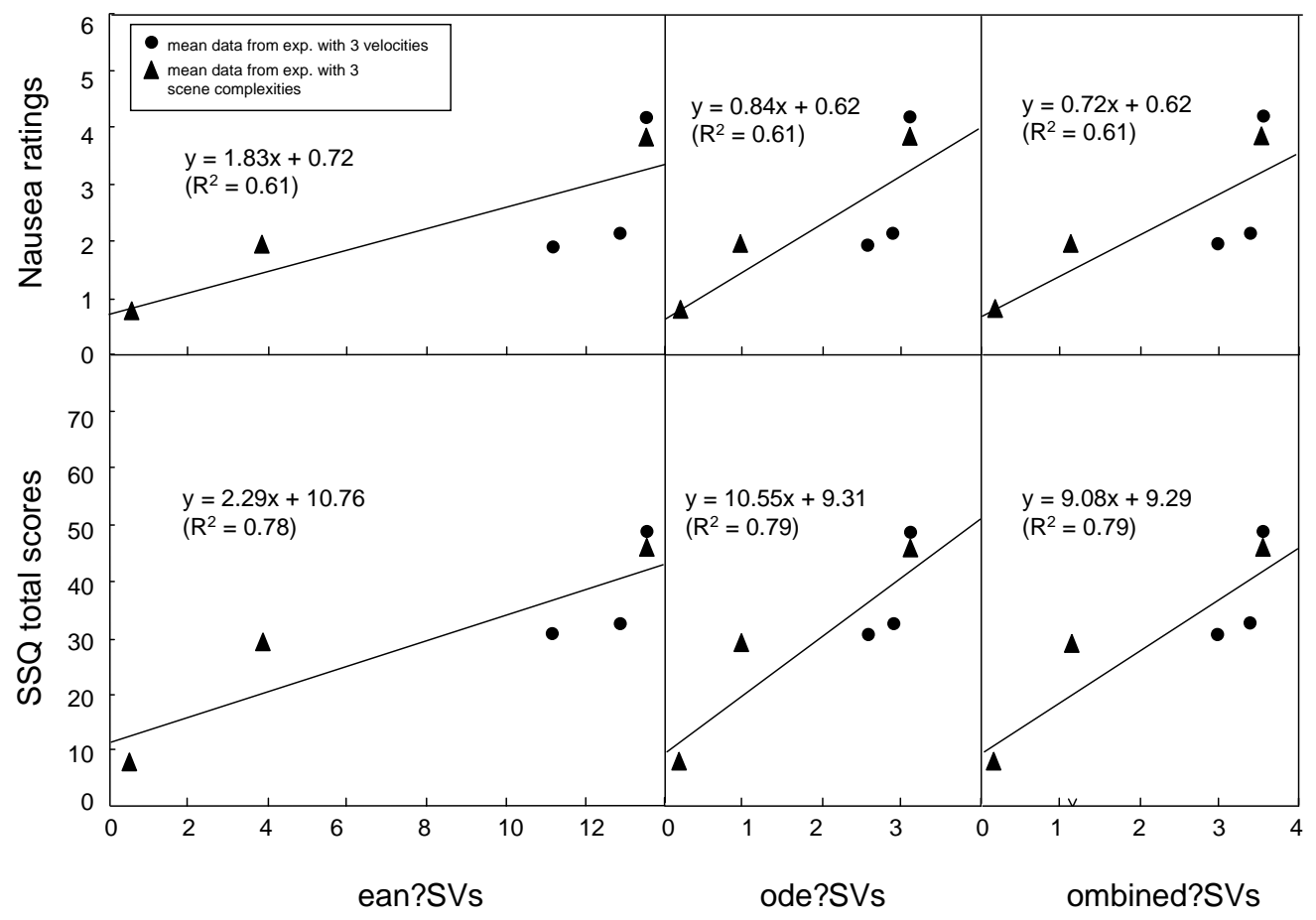

Figure 5. 


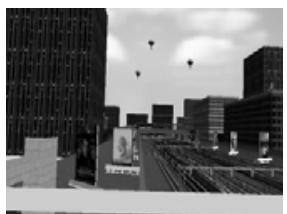

Radius $=\mathbf{0}$ (i.e., no blur) SF_horiz. SF_vert. $0.84 \mathrm{cpd} \quad 0.35 \mathrm{cpd}$ $0.05 \mathrm{cpd} \quad 0.06 \mathrm{cpd}$ $0.06 \mathrm{cpd} \quad 0.07 \mathrm{cpd}$

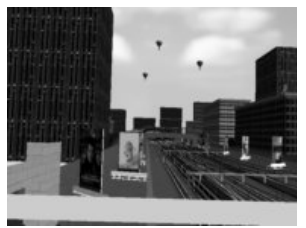

Radius $=1$

SF horiz. SF vert.

$0.26 \mathrm{cpd} \quad 0.22 \mathrm{cpd}$

$0.05 \mathrm{cpd} \quad 0.05 \mathrm{cpd}$

$0.06 \mathrm{cpd} \quad 0.06 \mathrm{cpd}$

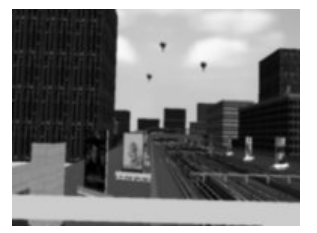

Radius $=2$

SF horiz. SF vert.

$0.15 \mathrm{cpd} \quad 0.18 \mathrm{cpd}$

$0.05 \mathrm{cpd} \quad 0.05 \mathrm{cpd}$

$0.06 \mathrm{cpd} \quad 0.06 \mathrm{cpd}$

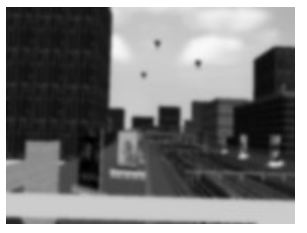

Radius $=\mathbf{4}$

SF horiz. SF vert. $0.09 \mathrm{cpd} \quad 0.1 \overline{6} \mathrm{cpd}$ $0.04 \mathrm{cpd} \quad 0.04 \mathrm{cpd}$ $0.06 \mathrm{cpd} \quad 0.05 \mathrm{cpd}$

Figure 6. 


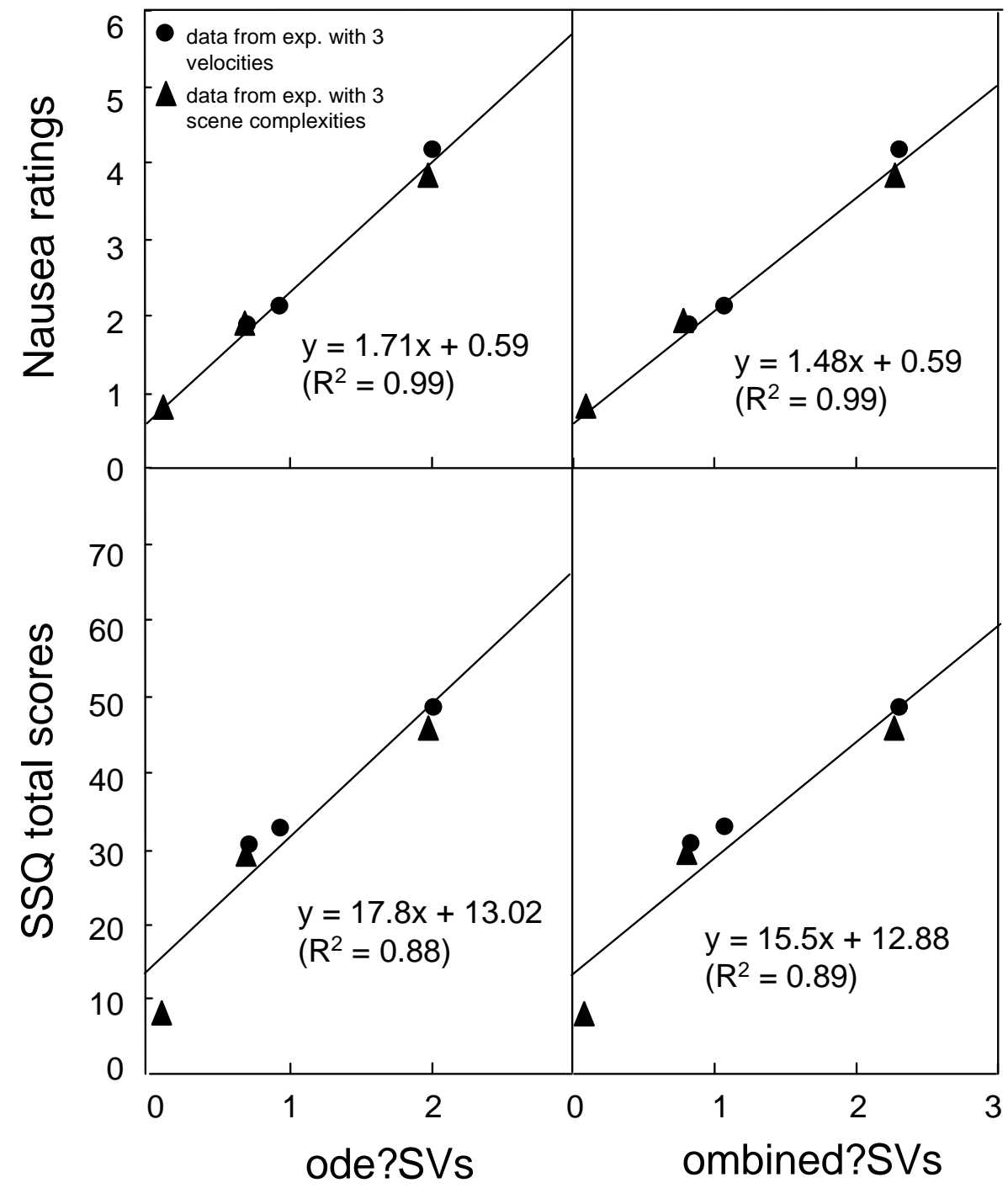

Figure 7 